\title{
Erratum: Local resolution of the Hubble tension: The impact of screened fifth forces on the cosmic distance ladder [Phys. Rev. D 100, 043537 (2019)]
}

\author{
Harry Desmond@, Bhuvnesh Jain, and Jeremy Sakstein
}

(Received 15 March 2020; accepted 18 March 2020; published 30 March 2020)

DOI: 10.1103/PhysRevD.101.069904

There was an error in our method for deriving constraints on $\Delta G / G_{\mathrm{N}}$ from the Cepheid-TRGB distance comparison in Sec. IV B. Correcting this, the bounds on $\Delta G / G_{\mathrm{N}}$ that we present in that section, Fig. 5, and Table IV strengthen by a factor of a few. Simultaneously, we learned that this test may be prone to systematic errors that we are unable directly to account for.

The test is unlikely to be statistics limited because it suffers from a number of potentially significant systematic uncertainties that are difficult to assess. The measurements in NED-D incorporate a diverse range of separate studies with different assumptions for the photometric pipeline, the metallicities of the Cepheids, the intrinsic and external extinction, and reddening and the zero-points for calibrating the Cepheid and TRGB distances. In addition, multiple studies may reduce the same data in different ways, so that the listed measurements are not independent. It is beyond the scope of our work to assess the robustness of this test in detail or attempt to mitigate the systematics. Further discussion of these and related issues may be found in [1-5], and we thank Rachael Beaton for explaining them to us.

In light of this, we now use the $5 \sigma$ limiting values of $\Delta G / G_{\mathrm{N}}$ from this test in our $H_{0}$ models, rather than $2 \sigma$ as previously. These $\Delta G / G_{\mathrm{N}}$ values are similar to what we used before due to the strengthening of the constraints, so that the impact of our screening framework on $H_{0}$, and hence the amount by which it reduces the Hubble tension, is only minorly affected. It is in fact slightly reduced, and we revise the statement that our models can achieve $\sim 1.5 \sigma$ consistency with Planck to $\sim 2 \sigma$.

The modified versions of Figs. 5, 6, 7 (here Figs. 1-3) and Table IV (here Table I) are shown below. In the future, it will be useful to investigate in detail the systematic uncertainties associated with the Cepheid-TRGB distace test. This would
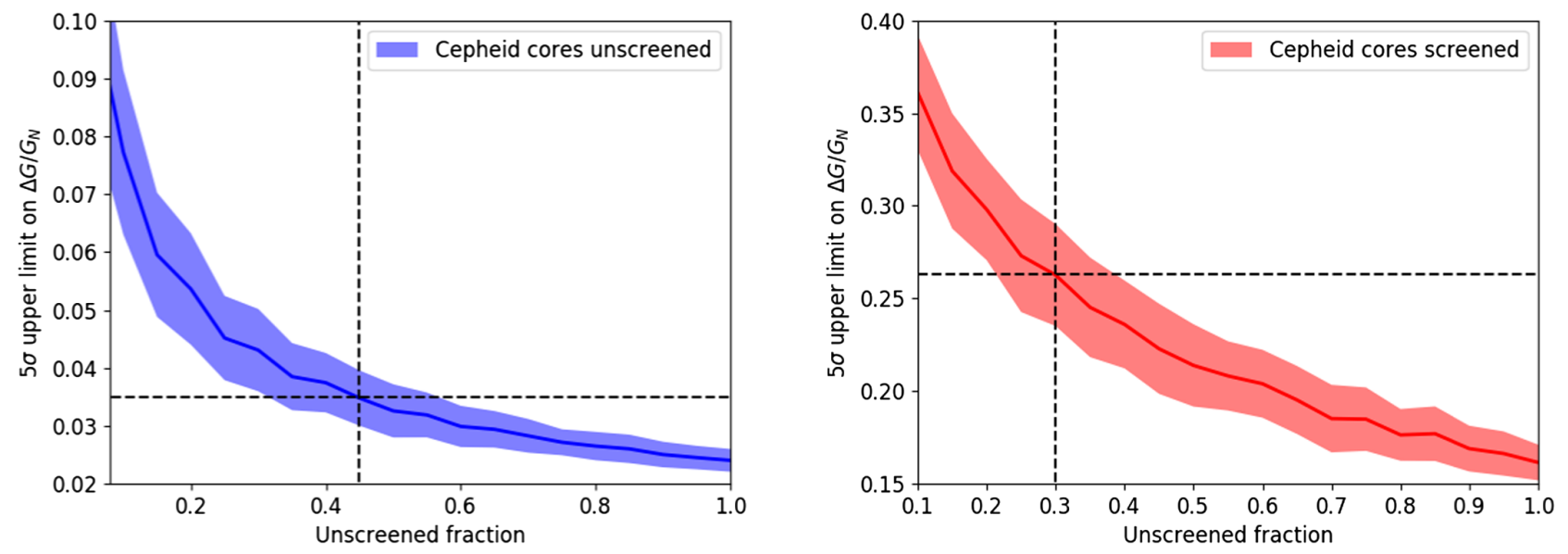

FIG. 1. Upper limit on $\Delta G / G_{\mathrm{N}}$ from the comparison of Cepheid and TRGB distance estimates to 51 galaxies from NED-D, as a function of the fraction of unscreened galaxies. We show separately the cases of Cepheids entirely unscreened (left) and only Cepheids' envelopes unscreened (right). Dashed lines indicate typical unscreened fractions as shown in Table I. 

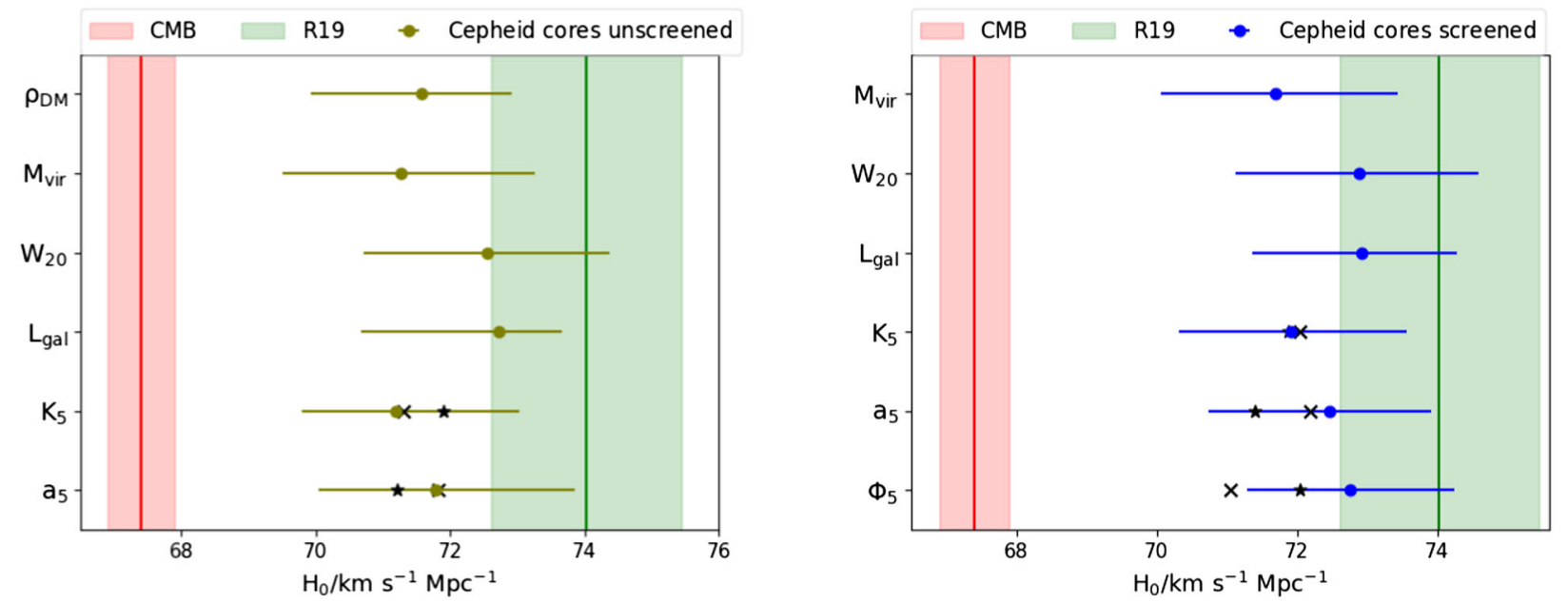

FIG. 2. Reconstructed $\hat{H}_{0}$ values and their uncertainties for the two main screening scenarios that we consider. The points in the left panel correspond to Cepheids entirely unscreened, and in the right panel to only Cepheid envelopes unscreened. In all cases we use the $5 \sigma$ maximum values for $\Delta G / G_{\mathrm{N}}$ from the Cepheid vs TRGB distance test (Sec. IV B and Fig. 1), which span the range $0.03-0.07$ for the left panel and $0.18-0.38$ for the right (Table I). The red and green vertical lines and shaded regions show the best-fit $H_{0}$ value, and its $1 \sigma$ uncertainty, from Planck and R19 respectively. For the environmental proxies $\Phi, a$ and $K$ we show results for a 5 Mpc aperture, but indicate also the result of using a 0.5 or $50 \mathrm{Mpc}$ aperture with black crosses and stars respectively (the error bars are similar). Note that the $\rho_{\mathrm{DM}}$ model must unscreen Cepheid cores so appears only in the left panel, while the converse is true for $\Phi$ assuming thin-shell screening.

TABLE I. The effect of various screening proxies on the value of $H_{0}$ inferred from the local distance ladder, assuming a $\Delta G / G_{\mathrm{N}}$ at the $5 \sigma$ limit of that allowed by the Cepheid-TRGB distance test, separately for each proxy. The first row indicates the R19 result. In the third column, " 1 " indicates unscreened (feels fifth force) and " 0 " screened for Cepheid envelope/Cepheid core/Calibration SNe/Cosmological $\mathrm{SNe}$. As described in the text, the threshold value for screening under a given proxy is given by the MW's value for that proxy. $\sigma_{H_{0}}$ is the discrepancy of the resulting $H_{0}$ constraint with the CMB. "E. U." denotes the "early unscreening" model (Appendix B) in which cosmological SNe are fully unscreened and calibration SNe fully screened, without any effect on Cepheids. In this case we consider $\Delta G / G_{\mathrm{N}}=0.05$. Our best models achieve $\sim 2 \sigma$ consistency with Planck.

\begin{tabular}{|c|c|c|c|c|c|c|}
\hline Proxy & Unscreened fraction & Screening properties & $\Delta G / G_{\mathrm{N}}$ & $\hat{H}_{0} / \mathrm{km} \mathrm{s}^{-1} \mathrm{Mpc}^{-1}$ & $\Delta \hat{H}_{0} / \mathrm{km} \mathrm{s}^{-1} \mathrm{Mpc}^{-1} / \mathrm{km} \mathrm{s}^{-1} \mathrm{Mpc}^{-1}$ & $\sigma_{H_{0}}$ \\
\hline$\ldots$ & $\ldots$ & $0 / 0 / 0 / 0$ & 0 & 74.0 & 1.4 & 4.4 \\
\hline$\Phi_{0.5}$ & 0.85 & $1 / 0 / 0 / 0$ & 0.18 & 71.1 & 1.4 & 2.5 \\
\hline$\Phi_{5}$ & 0.08 & $1 / 0 / 0 / 0$ & 0.38 & 72.8 & 1.5 & 3.4 \\
\hline \multirow[t]{2}{*}{$\Phi_{50}$} & 0.30 & $1 / 0 / 0 / 0$ & 0.26 & 72.0 & 1.6 & 2.8 \\
\hline & & $1 / 0 / 0 / 0$ & 0.26 & 72.2 & 1.6 & 2.9 \\
\hline$a_{0.5}$ & 0.31 & $1 / 1 / 0 / 0$ & 0.04 & 71.8 & 1.7 & 2.5 \\
\hline \multirow{2}{*}{ a5 } & & $1 / 0 / 0 / 0$ & 0.26 & 72.5 & 1.7 & 2.8 \\
\hline & 0.31 & $1 / 1 / 0 / 0$ & 0.04 & 71.8 & 1.9 & 2.3 \\
\hline \multirow[b]{2}{*}{$a_{50}$} & 035 & $1 / 0 / 0 / 0$ & 0.25 & 71.4 & 1.5 & 2.5 \\
\hline & 0.35 & $1 / 1 / 0 / 0$ & 0.04 & 71.2 & 1.6 & 2.3 \\
\hline \multirow{2}{*}{$K_{0.5}$} & 039 & $1 / 0 / 0 / 0$ & 0.24 & 72.0 & 1.5 & 2.9 \\
\hline & 0.39 & $1 / 1 / 0 / 0$ & 0.04 & 71.3 & 1.5 & 2.4 \\
\hline \multirow{2}{*}{$K_{5}$} & & $1 / 0 / 0 / 0$ & 0.26 & 71.9 & 1.5 & 2.8 \\
\hline & 0.30 & $1 / 1 / 0 / 0$ & 0.04 & 71.2 & 1.6 & 2.3 \\
\hline \multirow{2}{*}{$K_{50}$} & & $1 / 0 / 0 / 0$ & 0.27 & 71.9 & 1.5 & 2.8 \\
\hline & 0.20 & $1 / 1 / 0 / 0$ & 0.04 & 71.9 & 1.6 & 2.7 \\
\hline \multirow{2}{*}{$L_{\mathrm{gal}}$} & 012 & $1 / 0 / 0 / 0$ & 0.34 & 72.9 & 1.4 & 3.6 \\
\hline & 0.12 & $1 / 1 / 0 / 0$ & 0.07 & 72.7 & 1.5 & 3.5 \\
\hline \multirow{2}{*}{$W_{20}$} & 020 & $1 / 0 / 0 / 0$ & 0.30 & 72.9 & 1.7 & 3.0 \\
\hline & 0.20 & $1 / 1 / 0 / 0$ & 0.05 & 72.6 & 1.9 & 2.6 \\
\hline \multirow{2}{*}{$M_{\text {vir }}$} & 037 & $1 / 0 / 0 / 0$ & 0.24 & 71.7 & 1.7 & 2.4 \\
\hline & 0.31 & $1 / 1 / 0 / 0$ & 0.04 & 71.3 & 1.8 & 2.0 \\
\hline \multirow[b]{2}{*}{$\rho_{\mathrm{DM}}$} & & $1 / 1 / 0 / 0$ & 0.03 & 71.6 & 1.5 & 2.6 \\
\hline & 0.45 & $1 / 1 / 1 / 0$ & 0.03 & 72.0 & 1.5 & 2.9 \\
\hline E. U. & 1 & $0 / 0 / 0 / 1$ & 0.05 & 71.4 & 1.4 & 2.7 \\
\hline
\end{tabular}




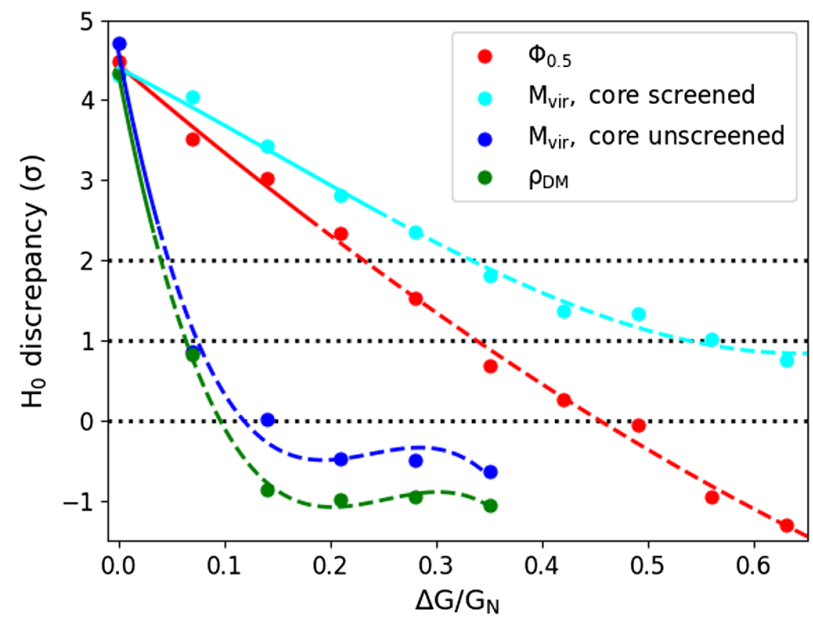

FIG. 3. Distance ladder vs CMB $H_{0}$ discrepancy $\sigma_{H_{0}}$ as a function of $\Delta G / G_{\mathrm{N}}$ for four screening models: $\rho_{\mathrm{DM}}, \Phi_{0.5}$, and $M_{\text {vir }}$ both with and without Cepheid luminosity modification. The lines are cubic fits to the corresponding points. We cut the $\rho_{\mathrm{DM}}$ and $M_{\mathrm{vir}}$ models where they are inconsistent with all the tests of Sec. IV B, and dash the lines where they enter $5 \sigma$ tension with the Cepheid vs TRGB distance test.

reveal whether our $5 \sigma$ limits are overly lenient so that our $H_{0}$ constraints are overoptimistic, or conversely whether even larger $\Delta G / G_{\mathrm{N}}$ values are allowed.

[1] I. S. Jang, D. Hatt, R. L. Beaton, M. G. Lee, W. L. Freedman, B. F. Madore, T. J. Hoyt, A. J. Monson, J. A. Rich, V. Scowcroft, and M. Seibert, Astrophys. J. 852, 60 (2018).

[2] D. Hatt, R. L. Beaton, W. L. Freedman, B. F. Madore, I.-S. Jang, T. J. Hoyt, M. G. Lee, A. J. Monson, J. A. Rich, V. Scowcroft, and M. Seibert, Astrophys. J. 845, 146 (2017).

[3] R. L. Beaton, G. Bono, V. F. Braga, M. Dall’Ora, G. Fiorentino, I. S. Jang, C. E. Martínez-Vázquez, N. Matsunaga, M. Monelli, J. R. Neeley, and M. Salaris, arXiv:1808.09191.

[4] R. L. Beaton, M. Seibert, D. Hatt, W. L. Freedman, T. J. Hoyt, I. S. Jang, M. G. Lee, B. F. Madore, A. J. Monson, J. R. Neeley, J. A. Rich, and V. Scowcroft, Astrophys. J. 885, 141 (2019).

[5] W. L. Freedman et al., Astrophys. J. 882, 34 (2019). 